\title{
Isoprostanes and Neuroprostanes as Biomarkers of Oxidative Stress in Neurodegenerative Diseases
}

\author{
Elżbieta Miller, ${ }^{1,2}$ Agnieszka Morel, ${ }^{3}$ Luciano Saso, ${ }^{4}$ and Joanna Saluk ${ }^{3,5}$ \\ ${ }^{1}$ Department of Physical Medicine, Medical University of Lodz, Hallera 1, Lodz, Poland \\ ${ }^{2}$ Neurorehabilitation Ward, III General Hospital in Lodz, Milionowa 14, Lodz, Poland \\ ${ }^{3}$ Department of General Biochemistry, Faculty of Biology and Environmental Protection, University of Lodz, \\ Pomorska 141/143, 90-236 Lodz, Poland \\ ${ }^{4}$ Department of Physiology and Pharmacology "Vittorio Erspamer", Sapienza University of Rome, Rome, Italy \\ ${ }^{5}$ Department of Toxicology, Faculty of Pharmacy with Division of Medical Analytics, Wroclaw Medical University, \\ Borowska 211, 50-556 Wroclaw, Poland
}

Correspondence should be addressed to Elżbieta Miller; betty.miller@interia.pl

Received 12 February 2014; Revised 28 March 2014; Accepted 31 March 2014; Published 29 April 2014

Academic Editor: Kota V. Ramana

Copyright (C) 2014 Elżbieta Miller et al. This is an open access article distributed under the Creative Commons Attribution License, which permits unrestricted use, distribution, and reproduction in any medium, provided the original work is properly cited.

\begin{abstract}
Accumulating data shows that oxidative stress plays a crucial role in neurodegenerative disorders. The literature data indicate that in vivo or postmortem cerebrospinal fluid and brain tissue levels of $\mathrm{F}_{2}$-isoprostanes $\left(\mathrm{F}_{2}\right.$-IsoPs) especially $\mathrm{F}_{4}$-neuroprotanes $\left(\mathrm{F}_{4}\right.$ NPs) are significantly increased in some neurodegenerative diseases: multiple sclerosis, Alzheimer's disease, Huntington's disease, and Creutzfeldt-Jakob disease. Central nervous system is the most metabolically active organ of the body characterized by high requirement for oxygen and relatively low antioxidative activity, what makes neurons and glia highly susceptible to destruction by reactive oxygen/nitrogen species and neurodegeneration. The discovery of $\mathrm{F}_{2}$-IsoPs and $\mathrm{F}_{4}$-NPs as markers of lipid peroxidation caused by the free radicals has opened up new areas of investigation regarding the role of oxidative stress in the pathogenesis of human neurodegenerative diseases. This review focuses on the relationship between $\mathrm{F}_{2}$-IsoPs and $\mathrm{F}_{4}$-NPs as biomarkers of oxidative stress and neurodegenerative diseases. We summarize the knowledge of these novel biomarkers of oxidative stress and the advantages of monitoring their formation to better define the involvement of oxidative stress in neurological diseases.
\end{abstract}

\section{Introduction}

The CNS (central nervous system) is very vulnerable to oxidative injury due to its high oxygen demand, high level of polyunsaturated fatty acids (PUFAs), and weak antioxidant defenses. The vulnerability of the brain to oxidative damage increases with the age due to reduced integrity of the bloodbrain barrier (BBB) and increased mitochondrial dysfunction [1-19]. Brain aging and neurodegeneration are characterized by chronic inflammation with persistent microglial activation and higher level of proinflammatory cytokines [4]. In addition, it promotes oxidative stress and neuronal damage. Neurons are particularly vulnerable to oxidative damage not only due to excitotoxicity but also to mitochondrial dysfunction. Moreover, neuronal membranes have plenty of unsaturated fatty acids. At higher concentrations, reactive oxygen/nitrogen species (ROS/RNS) cause neural membrane damage. Therefore, it can change not only membrane fluidity but also decreased activities of membrane-bound enzymes, ion channels, and receptors. The main sources of ROS/RNS are the mitochondrial respiratory chain, an uncontrolled arachidonic acid (AA) cascade, and NADPH oxidase (nicotinamide adenine dinucleotide phosphate-oxidase) $[5,6]$.

It is known that both inflammation and oxidative stress contribute to the development of various neuropathologies including Alzheimer's disease (AD), Parkinson's disease (PD), and multiple sclerosis (MS) $[5,6,20]$. As discussed by Guest et al. [7] the cerebrospinal fluid (CSF) of participants aged over 45 years contained statistically higher amounts of the oxidative damage marker $\mathrm{F}_{2}$-isoprostane $\left(\mathrm{F}_{2}\right.$-IsoPs $)$ and the inflammatory cytokine IL-6.

Brain response to oxidative stress-mediated neurodegeneration is very complex. All brain structures are involved 
in this multifactorial process. Astrocytes constitute approximately $90 \%$ of human brain and protect neurons from excitotoxicity through glutamate uptake system, and on the other side astrocytes contribute to the extracellular glutamate via reversed glutamate transporter [5]. Moreover, they may undergo astrocytosis after dopaminergic cell loss and are involved in the inflammatory processes. In general, inflammation is a protective response. The main mediators of neuroinflammation are microglial cells. Microglial cells consist mainly of macrophages and react to oxidative stress by transformation into activated microglia that are characterized by amoeboid morphology and rapid migration. Chronic activation of microglia may cause neuronal damage through the release of potentially toxic molecules such as proinflammatory cytokines, matrix metalloproteinases, ROS/RNS, proteinases, prostaglandin $\mathrm{E}_{2}$, complement proteins, and growth factors and also leads to the DNA and RNA damage $[5,21]$. These factors have neuroprotective properties but on the other hand they can be responsible for acceleration of oxidative stress and neurodegeneration.

Markers of lipid peroxidation include different molecules such as 4-hydroxy-trans-2-nonenal (4-HNE), 4-oxo-trans2-nonenal (4-ONE), acrolein, isoprostanes, and isofurans. These markers are derived from AA, which is released from neural membrane glycerophospholipids through the activation of cytosolic phospholipases $\mathrm{A}_{2}\left(\mathrm{cPLA}_{2}\right)$ [22].

Lipid peroxidation is a hallmark of oxidative stress. High level of lipid peroxidation products is a characteristic for many human diseases, especially neurodegenerative diseases. It can cause damage to cellular membranes through changes of membrane organization and alteration of membrane integrity, fluidity, and permeability [23].

\section{Biomarkers}

Biomarkers are defined as the indicators of normal biological processes or pathologic processes that can be objectively measured and evaluated [1]. The well-characterized, appropriate biomarkers may be used for health examination, diagnosis of pathologic processes at early stage, assessment of treatment response, and prognosis. Noninvasive measurements of circulating levels of specific biomarker are useful along the whole spectrum of the disease process and before diagnosis biomarkers could be used for screening and risk assessment of the diseases [2]. Moreover, biomarkers may be used for precise measurement of oxidative stress status in vivo [3]. Among the biological molecules, lipids appear to be the most susceptible to the attack of ROS/RNS [24, 25] and lipid peroxidation has been implicated in the neurodegeneration [11]. Therefore, the levels of lipid peroxidation products may be used as a biomarker for the measurement of oxidative stress status in vivo in neurodegenerative diseases [3]. The levels of lipid peroxidation products in biological fluids and tissues of human subjects have been measured extensively [26]. Presently, various lipid peroxidation products are applied for assessment of lipid peroxidation and oxidative stress status in vivo.

The measurement of $\mathrm{F}_{2}$-IsoPs is currently the best available biomarker of lipid peroxidation $[8,10,22,24]$.

\section{The Isoprostanes Pathways}

In the mid-1970s, it was shown that PG-like compounds could be formed in vitro by the nonenzymatic peroxidation of purified PUFAs. $\mathrm{F}_{2}$-IsoPs have been discovered in 1990 by Milne et al. [27] and Roberts II and Morrow [28] and since then they collected a lot of evidence that these compounds might be biomarkers of lipid peroxidation and oxidative stress in vivo as well as in vitro.

$\mathrm{F}_{2}$-IsoPs are a unique prostaglandin-like products, which are formed via nonenzymatic, free radical-mediated peroxidation of polyunsaturated fatty acids-for example, AA $[28,29]$. The oxidation of AA proceeds by many competing reactions to give numerous products. IsoPs containing a variety of prostane ring structures are composed of various isomers including $\mathrm{F}_{2}$-IsoPs, which are isomeric to $\mathrm{PGF}_{2 \alpha}$ $[27,30,31]$ and $D_{2} / E_{2}$-IsoPs, which are isomers of $P_{G D}$ and $\mathrm{PGE}_{2}$, respectively [32]. The mechanism of $\mathrm{F}_{2}$-IsoPs formation involves several steps. In the first stage, ROS reacts with the arachidonic acid and undergoes abstraction of an bisallylic hydrogen atom to yield an arachidonyl carboncentered radical. What is more, there is insertion of oxygen, which leads to the formation of peroxyl radicals. Four different peroxyl radical isomers are formed depending on site of hydrogen abstraction and oxygen insertion. Peroxyl radicals isomers undergo 5-endocyclization and a second molecule of oxygen adds to the backbone of the compound to form four bicyclic endoperoxide intermediate regioisomers$\mathrm{PGH}_{2}$-like compounds. These unstable bicycloendoperoxide intermediates are reduced to the $\mathrm{F}_{2}$-IsoPs and four $\mathrm{F}_{2}$-IsoPs regioisomers are formed $[30,33,34]$. This regioisomers are reduced to four series of F-ring regioisomers (15-, 8-, 12-, 5 -series), each consisting of eight racemic diastereoisomers. These regioisomers are depending on the carbon atom to which the side chain hydroxyl is attached [8].

To sum up, the biosynthetic steps of IsoPs include formation of the following:

(i) three arachidonyl radicals,

(ii) four peroxyl radical isomers with subsequent endocyclization; finally, formation of bicycloendoperoxide regioisomers, which are reduced to $\mathrm{F}_{2}$-IsoPs; IsoPs are compounds that have F-type prostane rings isomeric to $\mathrm{PGF}_{2 \alpha}$.

The alternative ring structures, D/E-type and A/J-type prostane, are formed by the same mechanisms [32, 34]. So that E- or D-ring and thromboxane-ring compounds of IsoPs are formed during the rearrangement of isoprostane endoperoxides in vivo. $\mathrm{E}_{2}$ - and $\mathrm{D}_{2}$-IsoPs are not terminal products of the IsoP pathway. These compounds are unstable and readily undergo dehydration in vivo to yield $\mathrm{A}_{2} / \mathrm{J}_{2}$-IsoPs. The cyclopentenone IsoPs might be neurotoxic products of the IsoPs pathway and might contribute to the pathogenesis of oxidative neurodegeneration. $\mathrm{A}_{2}-/ \mathrm{J}_{2}$-IsoPs contain $\alpha, \beta$ unsaturated carbonyls, which rapidly adduct cellular thiols and these cyclopentenone IsoPs induce neuronal apoptosis and promote neurodegeneration [27].

The other electrophilic lipid peroxidation products can also damage neurons. The $\gamma$-ketoaldehydes (e.g., isoketals, 
isolevuglandins), highly reactive acyclic compounds, might be formed as a products of IsoPs endoperoxide rearrangement [35].

PUFAs are the most susceptible to free radical attack and, in general, oxidizability increases as the number of double bonds increases. So, the oxidizability of PUFAs can be estimated by the linear increase in the rate of oxidation with the increasing number of active methylene groups located between two bonds. From such correlation, the oxidizability of each PUFA is increased for about twofold for each active methylene group. Thus, the oxidizability of common fatty acids is as follows: linoleic acid $(18: 2)<$ arachidonic acid $(20: 4, n-6)<$ eicosapentaenoic acid (EPA, $20: 5, n-3)<$ docosahexaenoic acid (DHA, $22: 6, n-3)[27,36]$.

The oxidation mechanisms of IsoPs are well known, but they are not the only substrate for the IsoPs pathway. The presence of at least three double bonds in fatty acid molecule allows the cyclization.

F2-dihomo-isoprostanes (F2-dihomo-IsoPs) are the peroxidation products from adrenic acid, which is the main component of myelin. The great amount of DHA is observed in brain but primarily found in white matter and is associated with myelin. White matter is commonly damaged by ischemic stroke and is uniformly damaged in MS. F2dihomo-IsoPs are generated in significant amounts from adrenic acid and their levels are greatly increased in settings of oxidative stress occurring in the white matter portion of the human brains. Roberts II and Milne [8] demonstrate that, proportionally, levels of F2-dihomo-IsoPs in white matter undergoing oxidative injury increase to a greater extent than IsoPs and NeuroPs derived from AA and DHA, respectively. Their studies suggest that the quantification of F2-dihomoIsoPs might be a selective marker of white matter injury in vivo [8].

F2-dihomo-IsoPs are also present in kidney, adrenal glands, and tissues and might be regarded as an early marker of lipid peroxidation in Rett syndrome-a disorder of the nervous system that leads to developmental reversals, especially in the areas of expressive language and hand use [37].

3.1. AA Is Not the Only One PUFA That Can Be Oxidized to Form IsoPs. By the peroxidation of the $\omega$-3 PUFA, EPA and DHA, F-ring IsoPs have been generated. The IsoPs-like compounds generated from this acid are named NeuroPs [8].

$\mathrm{F}_{3}$-IsoPs are formed in abundance in vitro and in vivo from EPA nonenzymatically peroxidation [38-40], while DHA may be oxidized nonenzymatically into $\mathrm{F}_{4^{-}}, \mathrm{D}_{4^{-}}, \mathrm{E}_{4^{-}}$ , $\mathrm{A}_{4^{-}}$, and $\mathrm{J}_{4}$-neuroprostanes $\left(\mathrm{F}_{4^{-}}, \mathrm{D}_{4^{-}}, \mathrm{E}_{4^{-}}, \mathrm{A}_{4^{-}}\right.$, and $\mathrm{J}_{4^{-}}$ NeuroPs) $[38,39]$. AA is relatively evenly distributed in brain with similar concentrations in gray matter and white matter, and within glia and neurons. Unlike AA, DHA is highly concentrated in neuronal membranes to the exclusion of other cell types. Moreover, $\mathrm{F}_{4}$-NeuroPs are by far the most abundant products of this pathway in the brain [32]. The quantification of $\mathrm{F}_{4}$-NeuroPs provides a highly selective quantitative window for neuronal oxidative damage in vivo. Thus, $\mathrm{F}_{2}$-IsoPs quantification is a reflection of oxidative damage to the brain in general and $\mathrm{F}_{4}$-NeuroPs in particular [40,41]. Roberts II and Milne [8] have found that the level of IsoPs produced from the oxidation of EPA significantly exceeds those of the $\mathrm{F}_{2}$-IsoPs generated from AA. This is because EPA contains more double bonds, and therefore, it is more easily oxidizable. The authors have also observed that EPA supplementation markedly reduced levels of arachidonate-delivered $\mathrm{F}_{2}$-IsoPs mouse heart tissues by over $60 \%$. Such observations are crucial because $\mathrm{F}_{2}$-IsoPs are generally considered as a proinflammatory molecules associated with the pathophysiological sequelae of oxidant stress. It is thus surprising to propose that the part of mechanism by which EPA prevents certain diseases is its ability to decrease $\mathrm{F}_{2}$-IsoP generation [8].

3.2. IsoPs As Biomarkers of Lipid Peroxidation in Neurodegenerative Diseases. Oxidative stress is caused by an imbalance between free radicals production and antioxidant defenses in favor of the oxidation and leads to lipid peroxidation, membrane protein, and DNA damage and is thought to be important in the pathogenesis of a variety of neurological disorders, especially neurodegenerative diseases or atherosclerosis, cancer, and aging [42]. Lipid peroxidation is the most important source of free radical-mediated injury that directly damages neuronal membranes and yields a number of secondary products responsible for extensive cellular damage. Any specific repair process of lipid peroxidation does not exist as it does for proteins and DNA and this may explain why moderate levels of lipid peroxidation could have physiological significance for cell signaling and membrane remodeling [7]. One of the major targets of the lipid peroxidation process is the CNS. The brain is the most susceptible to oxidative damage because of the high oxygen consumption, the low levels of antioxidant enzymes (catalase and glutathione peroxidase), the elevated levels of iron (a potent catalyst for oxidant formation), and the ability to oxidize different substrates (e.g., membrane polyunsaturated fatty acids). Despite the fact that free radicals can attack many various critical biological molecules, such as DNA and cellular proteins, the high content of unsaturated lipids renders lipid peroxidation, the central feature of oxidant injury in the brain [43]. Peroxidation of membrane lipids affects neuronal homeostasis resulting in augmented membrane inflexibility, diminished activity of membrane-bound enzymes (e.g., sodium pump), destruction of membrane receptors, and changed permeability $[44,45]$. One leading hypothesis is that the free radical-mediated oxidation of lipids contributes to the main pathological effects of oxidative stress in the brain. In support of this theory, increased levels of bioactive lipid peroxidation products have been identified in affected brain regions from humans with various neurodegenerative diseases [46, 47], as well as in corresponding animal models [48].

Due to the fact that free radicals are unstable and highly reactive, there are difficulties in direct measurement of their level. That is why elucidation of the importance of oxidative damage in neurological diseases is very hard. Because of their stability, the measurement of $\mathrm{F}_{2}$-IsoPs by mass spectrometry has been extensively employed as a marker of oxidant stress and is widely considered to be the gold-standard index of lipid peroxidation in vivo $[49,50]$. IsoPs can be relatively easily 
TABLE 1: Isoprostanes as markers of oxidative stress in neurodegenerative diseases.

\begin{tabular}{|c|c|c|c|c|c|}
\hline Classes of isoprostanes & Material & Disease & Study & Versus control & Reference \\
\hline \multirow{3}{*}{ F2-IsoPs } & CSF $^{*}$, post & Alzheimer disease & vivo & High & {$[10-13,32]$} \\
\hline & mortem brain & Creutzfeldt-Jakob & vivo/vitro & High & {$[17,18,53]$} \\
\hline & tissue, plasma, urinary & Huntington disease & vivo & High & {$[54]$} \\
\hline \multirow{3}{*}{ 8-iso PGF 2alfa } & Urine & SPMS $^{* *}$ & vivo & 6-fold & {$[55]$} \\
\hline & \multirow{2}{*}{ CSF } & $\mathrm{RRMS}^{* * *}$ & vivo & Higher & {$[9,53,56,57]$} \\
\hline & & ALS $^{* * * *}$ & vivo & Higher & {$[5,19,58]$} \\
\hline
\end{tabular}

$\mathrm{CSF}^{*}$ : cerebrospinal fluid; SPMS** ${ }^{*}$ secondary-progressive type of multiple sclerosis; RRMS***: relapsing-remitting type of multiple sclerosis. ALS****: amyotrophic lateral sclerosis.

quantified in body fluids because they are commonly found in urine, blood, and CSF and are also present in the exhaled air (Table 1). Their formation in vivo can be reliably monitored in every biological fluid by the noninvasive measurements of specific signals of lipid peroxidation, which tend to be sensitive and specific [51]. The measurement of $\mathrm{F}_{2}$-IsoPs has emerged as one of the most reliable approaches to assess oxidative stress status in vivo, providing an important tool to explore the role of oxidative stress in the pathogenesis of human disease. In the oxidative tissue injury the level of $\mathrm{F}_{2}$-IsoP is significantly increasing. The rapid development of analytical methods for IsoPs measurement helped clarify the role of the free radicals in human physiology and pathophysiology [52].

Measurement of $\mathrm{F}_{4}-\mathrm{NPs}$, the stable product of free radical damage to DHA, also provides valuable data in exploring the role of oxidative stress in neurodegenerative diseases. The products of the IsoP pathway were found to have strong biological actions and therefore may participate as physiological mediators of the disease [59]. Research on brain-derived IsoPs has begun only a few years ago, but it has already provided convincing evidence on the usefulness of these markers in understanding the role of oxidative damage in brain diseases [60]. IsoPs as active products of free-radicalmediated peroxidation of AA contained in phospholipids of cell membranes and lipoproteins have a potential relevance to human neurodegenerative and demyelinating diseases. The role of free radical-induced oxidative damage in the pathogenesis of neurodegenerative disorders has been definitely established [61-65]. The elevated formation of $\mathrm{F}_{2}$-IsoPs has been observed in brain tissues and body fluids in numerous neurodegenerative diseases, including Alzheimer's disease [32], Parkinson's disease [6], Huntington's disease (HD) [66], Creutzfeldt-Jakob disease (CJD) [66], multiple sclerosis [55], and amyotrophic lateral sclerosis (ALS) [43].

The measurement of free $\mathrm{F}_{2}$-IsoPs in plasma or urine can be utilized to assess the endogenous formation of IsoPs but not to reveal the organ in which they are formed. Determining the levels of IsoPs in the unique fluid compartmentCSF, which reflects the ongoing metabolic activity of the brain, provides a great opportunity to reveal the occurrence of oxidative stress and lipid peroxidation in the brain $[10,44]$.

\section{Multiple Sclerosis}

Multiple sclerosis is a multifactorial, heterogeneous disease with several pathophysiological components: inflammation, demyelination, redox, axonal damage, and repair processes. These components are not uniformly contributed in patient populations but can individually predominate [67, 68]. MS is a leading cause of neurological disabilities in young adults and affects up to $2.4 \%$ of population in USA and Canada and up to $1.9 \%$ in some European countries. It is considered to be autoimmune, or at least its etiopathogenesis involves intensive autoaggressive immune response [69]. MS is heterogeneous disease on several grounds. There are several different clinical courses of this disorder. The most usual (over $80 \%$ ) is relapsing-remitting course (RRMS) in which relapse occurs from time to time followed up by complete or partial recovery [67]. This stage of disease is characterized with multifocal inflammation, oedema, and cytokines actions. About half of RRMS patients after 10-20 years of disease lasting accumulate irreversible neurological deficits $[67,70]$. This type of MS is known as secondary progressive (SPMS) that is dominated by neurodegeneration processes and progression of clinical symptoms [71]. The next $20 \%$ of MS patients with progressive symptoms from the onset have primary-progressive (PPMS) type. For the transition from RRMS to progressive stage axonal injury is responsible [67]. Neurodegeneration of demyelinated axons is a major cause of irreversible neurological disability in MS. Disability levels in progressive forms of MS patients often worsen despite a stable MRI T(2) (magnetic resonance) lesion burden [67]. The presence of oxidative stress in the absence of measurable inflammation could help explain this phenomenon [3, 47].

Currently classifications of biomarkers of MS are connected with the pathophysiological processes. It has been divided into seven categories:

(1) alteration of the immune system (interleukins IL- $1 \beta$, IL-2, IL-4, IL-6, IL-10, IL-12, IL-23, interferon (IFN $\gamma$ ), tumor necrosis factor (TNF $\alpha)$, transforming growth factor (TGF $\beta)$, cytokines CXCR3/CXCL10-marker activated T cells; E-selectin, L-selectin, ICAM-1, VCAM-1, CD31, surface expression of LFA-1 and VLA-4 (adhesion molecules), CD40/CD40L, CD80, CD86, and heat shock proteins (hsp));

(2) axonal/neuronal damage (Tau protein, 24S-hydroxycholesterol, N-acetylaspartic acid);

(3) blood-brain barrier disruption (matrix metalloproteinases (MMPs): MMP-9 and their inhibitors (TIMP), platelet activating factor (PAF), and thrombomodulin); 
(4) demyelination (MBP and MBP-like material, proteolytic enzymes);

(5) oxidative stress and excitotoxicity (nitric oxide derivatives, $\mathrm{F}_{2}$-IsoPs, and uric acid);

(6) gliosis (glial fibrillary acid protein (GFAP), S-100 protein);

(7) remyelination and repair (NCAM (neural cell adhesion molecule), CNTF (ciliary neurotrophic factor), MAP-2 + 13 (microtubule-associated protein-2 exon 13), and CPK-BB (creatine phosphatase BB) [67].

IsoPs are the candidate biomarkers of lipid peroxidation in MS. In diseases with a complex pathogenesis an individual biomarker is reflected in only one of many ongoing pathogenic processes $[5,67]$. The data presented in our studies indicate that lipid peroxidation and oxidative stress in patients with MS may occur. It was found that the urine IsoPs level was over 6-fold elevated in patients with SPMS than in control [3]. The increased level of $15-\mathrm{F}_{2 t}$-IsoPs in CSF of MS patients has been described by Mattsson et al. [9]. To investigate the possible correlation between $\mathrm{F}_{2}$-IsoPs and the disease inflammatory activity it has been observed that the CSF levels of $15-\mathrm{F}_{2 t}$-IsoPs in patients with RRMS were not correlated with the clinical signs of the disease. These observations suggest that high levels of $\mathrm{F}_{2}$-IsoPs (about 9fold higher than control subjects) may represent an index of degenerative phenomena, which persist also in the lack of an ongoing inflammatory activity. In other researches, Minghetti et al. [10] have found that the CSF level of the reliable marker of oxidative stress in vivo, $15-\mathrm{F}_{2 t}$-IsoP, is 3 times higher in patients with MS than in a benchmark group of subjects with other neurologic diseases. This increase was not correlated with the $15-\mathrm{F}_{2 t}$-IsoP levels and was much lower in steroid-treated patients. Clearly, the levels of $15-\mathrm{F}_{2 t}-\mathrm{IsoP}$ were associated with the degree of disability. What is more, in the spinal cord of mice during early progressive stages of experimental autoimmune encephalomyelitis (EAE) the elevated levels of $\mathrm{F}_{2}$-IsoPs and $\mathrm{F}_{4}$-NPs were observed $[72,73]$. In white matter and myelin-forming oligodendrocytes the DHA levels are relatively low, which are affected in MS. So, $\mathrm{F}_{2}$-IsoPs might be preferable to $\mathrm{F}_{4}$-NPs as lipid peroxidation biomarkers in this demyelinating disease [66]. There are some studies which examine the correlation between levels of $\mathrm{F}_{2}$-IsoPs in CSF of MS patients, their healthy siblings, and unrelated controls. The CSF concentrations of $\mathrm{F}_{2}$-IsoPs in siblings of MS patients were significantly higher than in healthy controls. The $\mathrm{F}_{2}$-IsoPs levels in patients suffering from MS were intermediate between siblings, as well as controls. In patients with MS and siblings, the levels of $\mathrm{F}_{2}$ IsoPs were significantly correlated. These researches have been proved that siblings of MS patients have an increased oxidative stress response to the environmental and genetic factors that might be involved in MS pathogenesis [9].

\section{Alzheimer's Disease}

Alzheimer's disease (AD) is one of the major causes of dementia, which is characterized by the deposition of the amyloid $\beta(\mathrm{A} \beta)$ peptide and microtubule-associated protein tau in the brain $[74,75]$. The critical role in the $\mathrm{AD}$ pathogenesis plays an abnormal tau phosphorylation. It has been proved that $\mathrm{A} \beta$ has capacity to interact with transition metals generating redox active ions, which precipitate in lipid peroxidation and cellular oxidative stress [76]. In other words, $\mathrm{A} \beta$ promotes cellular oxyradicals accumulation in neurons and glial cells in vulnerable regions of $\mathrm{AD}$ brain. Such oxidative stress may lead to many of the metabolic and neurodegenerative alterations observed in this disease [77]. Moreover, in tau phosphorylation, the mediation of oxidant toxicity by $\mathrm{A} \beta$ has been also implicated. Besides the oxidative stress, the mitochondrial dysfunction has been observed in $\mathrm{AD}$ [78]. A variety of markers of oxidative stress are increased, with a clear relationship with $\mathrm{A} \beta$ deposition and neurofibrillary degeneration has been observed in postmortem brain tissues from $\mathrm{AD}$ patients [79]. It has been reported that the activity and/or protein levels of several antioxidant enzymes were altered in $\mathrm{AD}$ brain regions, consistent with ongoing oxidative stress [11]. Increased $\mathrm{F}_{2}$ IsoPs and $\mathrm{F}_{4}$-NPs levels in the postmortem ventricular fluid from definite $\mathrm{AD}$ patients had been firstly demonstrated by Montine et al. [11]. The authors, given the partial overlap between CSF concentrations of $\mathrm{F}_{2}$-IsoPs in $\mathrm{AD}$ patients and healthy subjects, suggested that the quantification of CSF $\mathrm{F}_{2}$-IsoPs could not be utilized as an early marker of dementia. There was no correlation between $\mathrm{CSF} \mathrm{F}_{2}$-IsoPs and age or duration of disease. This study concerns the relative small group of $\mathrm{AD}$ patients and probably may not be fully representative of the $\mathrm{AD}$ population [11]. In an independent study, Musiek and colleagues [80] demonstrated the formation of $\mathrm{F}_{4}$-NPs during peroxidation of DHA in vitro $\mathrm{F}_{4}-\mathrm{NPs}$ may be used as a marker of lipid peroxidation in the pathogenesis of neurodegenerative diseases, because in these diseases the elevated levels of $\mathrm{F}_{4}$-NPs is observed. Subsequently, they proved the presence of esterified $\mathrm{F}_{4}$-NPs in the human brain and showed abnormally high levels in occipital and temporal lobes of AD brains. Interestingly, while in vitro oxidation of DHA yields 3.4-fold higher levels of $\mathrm{F}_{4}$ NPs compared with $\mathrm{F}_{2}$-IsoPs, the CSF levels of these two classes of compounds showed a very close correlation in a small number of $\mathrm{AD}$ patients [66].

In Yao et al.'s [12] and Praticò et al.'s [13] researches, found that the contents of $15-\mathrm{F}_{2 t}$-IsoPs and $\mathrm{IPF}_{2}$ alpha-VI were markedly elevated in the frontal and temporal lobes of $\mathrm{AD}$ brains compared to the corresponding cerebella and to the same regions of control brains. Moreover, there was also a significant correlation between the levels of the two IsoPs measured in each $\mathrm{AD}$ brain. In postmortem ventricular CSF, $\mathrm{IPF}_{2}$ alpha-VI levels were higher in $\mathrm{AD}$ patients than in healthy people. In contrast, brains levels of 6-keto $\mathrm{PGF}_{1}$ alpha, an index of prostaglandin production, and ventricular CSF $15-\mathrm{F}_{2 t}$-IsoP levels did not differ in $\mathrm{AD}$ and control subjects.

$\mathrm{F}_{2}$-IsoPs were measured also in plasma and in urine of $\mathrm{AD}$ patients. It has been shown that plasma and urinary levels were higher than controls, but only in the case of plasma the difference was statistically significant. So, plasma or urine content of IsoP in patients with AD reflects a specific increase in oxidative stress within the brain or a more generalized 
systemic oxidative stress remains to be determined. The authors also found that in the control group $\mathrm{F}_{2}$-IsoPs levels in females were higher than in males and suggested that this could be related to an increase in oxidative stress associated with the loss of estrogens in the postmenopausal period [81]. Indeed, estrogens can be antioxidants because of their phenolic structure [82] or may upregulate apolipoprotein E, favoring the formation of the apolipoprotein E/A-complex, and thus the sequestration of $\mathrm{A} \beta$. Consistent with this hypothesis, Praticò et al. [13] demonstrated markedly elevated $\mathrm{F}_{2}$-IsoPs in the brains of aged apolipoprotein E-deficient mice compared with wild-type C5 [83].

\section{Huntington's Disease}

The abnormal expansions of an unstable cytosine-adenineguanine repeat region at the $5^{\prime}$-end of a gene on chromosome 4 are the main cause of this disease. This genetic abnormality results in the expression of an expanded polyglutamine tract in huntingtin protein, which can aggregate in neuronal nuclei and dystrophic neuritis in Huntington's disease brains. The HD gene defect causing the death of specific populations of striatal neurons is still unknown. The elevated oxidative damage observed in areas of degeneration in patients' brains with $\mathrm{HD}$ and the increased free radical production in animal models indicate the involvement of oxidative stress either as a cause or as a consequence of the cell death cascade in the disease $[66,84]$. There are a lot of studies suggesting that oxidative stress is prominent in the neostriatum of $\mathrm{HD}$ brains [85] and contributes to degeneration of the neostriatum. In patients suffering from $\mathrm{HD}$, the mitochondrial dysfunction results in overproduction of ROS leading to oxidative and nitrosative stress $[54,86-88]$. Such stress contributes to neuronal dysfunction by damaging the main structures: DNA, proteins, and lipids. It has been shown that the highly reactive product of nitric oxide and superoxide free radicalsperoxynitrite, which inhibits mitochondrial respiration and reduce antioxidant defenses in cells, is marked by increased of 3-nitrotyrosine (3-NT) levels [54, 87, 89]. The immunoreactivity of 3-NT is increased in postmortem HD brain tissue [85]. Also, increased levels of protein carbonyls in HD striatum and cerebral cortex have been observed [85]. It has been observed that 4-hydroxynonenal and malondialdehyde, lipid peroxidation products, are increased eightfold in HD human plasma [90] and also in postmortem brain tissue [84].

The measurement of the levels of $\mathrm{F}_{2}$-IsoPs in the CSF of $\mathrm{HD}$ patients indicates the contribution of oxidative stress to the pathogenesis of HD. The level of $\mathrm{F}_{2}$-IsoP in HD patients was significantly higher than in the control group. However, the overlap of levels between these groups suggested that the oxidative damage to the brain may not occur uniformly in the early phase of the disease. But like in $\mathrm{AD}$, correlation between $\mathrm{F}_{2}$-IsoPs and age or disease duration there was not found; moreover no difference between men and women was observed [66].

In addition, in HD plasma the glutathione levels are significantly reduced [91]. Browne and Beal [14] suggest that in transgenic HD mice, there are increased immunostaining for malondialdehyde, 4-hydroxynonenal, and $15-\mathrm{F}_{2 t}$-IsoPs [54].

\section{Creutzfeldt-Jakob Disease}

Creutzfeldt-Jakob disease (CJD) is one of the most known human transmissible spongiform encephalopathies (TSEs) or prion diseases, a heterogeneous group of infectious, sporadic, and genetic disorders characterized by rapidly progressive dementia. The characteristic neuropathological hallmark of the disease is the amyloid deposition of the pathological form of a cellular protein (like in $\mathrm{AD}-\mathrm{A} \beta$ or $\mathrm{HD}$-huntingtin). The accumulation of the pathological prion protein is considered as a central event and is thought to trigger several pathogenetic mechanisms, eventually culminating in the typical spongiform degeneration [66].

The physiological functions of cellular prion protein are still unknown; however, due to its cooper binding ability it might play an important role in the oxidative homeostasis of the brain and could act as an antioxidant. These antioxidant properties may be related to its superoxide dismutase- (SOD) like activity $[15,16]$. Kralovicova et al. [15] have proved that these cells, which express higher levels of prion protein, are more resistant to oxidative stress. Wong et al. suggest [16] that the levels of several oxidative stress markers, protein carbonyl groups and products of lipid peroxidation, were increased in brain tissues of prion protein knockout mice [92]. In brains of mice infected with scrapie, the elevated levels of nitrotyrosine and heme oxygenase- 1 had been found [93]. It has been suggested that the level of lipid peroxidation products is increased in brains of scrapie-infected mice and also prion proteins purified from brains of these animals possess a reduced SOD-like activity [94].

The increased levels of $\mathrm{F}_{2}$-IsoP in CSF of CreutzfeldtJakob patients have been observed in Minghetti et al's [17] researches. Also, another product of lipid peroxidation has been found to be unchanged in CSF from patients suffering from CJD in comparison to controls [95]. Arlt et al. [18] found that CSF lipids from patients suffering from CJD were more susceptible to oxidation process than those from nondemented controls. Thus, they observed that in the CJD patients, the levels of antioxidants and the amount of PUFAs were reduced. Their researches indicate that oxidative stress is elevated in CJD patients and the oxidative mechanisms are correlated with pathogenesis of this disease.

It has been observed that in patients with sporadic and familial CJD, CSF levels of $15-\mathrm{F}_{2 t}$-IsoP were about 2.5 -fold higher than in patients with noninflammatory disorders. No correlation was found between $15-\mathrm{F}_{2 t}$-IsoPs and $\mathrm{PGE}_{2}$ and also $15-\mathrm{F}_{2 t}-$ IsoP levels and age of patients nor polymorphism at codon 129 of the prion protein gene, indicating that lipid peroxidation and prostaglandin synthesis are unrelated phenomena in this disease. $\mathrm{PGE}_{2}$ concentrations, that were about 6.5-fold higher than in controls, were inversely correlated with patient survival; meanwhile, the levels of $15-\mathrm{F}_{2 t}$-IsoP were not correlated with the clinical duration of the disease. It has been suggested that the inflammation might be more relevant than oxidative stress to the pathogenesis of this particular disease $[53,66]$.

In other studies, it has been proved that the increased level of $\mathrm{PGE}_{2}$ in hippocampal is associated with a strong induction of COX-2 expression, which was elevated with 
progression of disease and is localized to microglial cells [56]. In sporadic CJD patients the shorter survival was associated with higher levels of $\mathrm{PGE}_{2}$ in CSF patients. $\mathrm{PGE}_{2}$ may be an index of disease severity rather than progression, because $\mathrm{PGE}_{2}$ levels were not dependent on the time of CSF sampling during the course of the disease [10]. $\mathrm{PGE}_{2}$ can be associated with neuronal death, because in neuroblastoma cells, prion proteins peptides increase $\mathrm{PGE}_{2}$ levels and COX-1 inhibitors protect against prion proteins toxicity [57]. Whether $\mathrm{PGE}_{2}$ contributes to neuronal death in CJD, is a consequence of neuronal apoptosis, or is just an index of the disease state remains to be established.

\section{Amyotrophic Lateral Sclerosis}

Amyotrophic lateral sclerosis (ALS) is a multifactorial and complex disease, in which genetic, environmental, or geneticenvironmental interactions lead to motor neuronal degeneration. The deposition of a misfolded protein in neural tissue, in this instance copper/zinc SOD, is characteristic for the ALS and other neurodegenerative diseases [96, 97]. Several neuroinflammatory changes, such as increased levels of proinflammatory molecules, astrogliosis, and also microglial activation, which are characteristic for many neurodegenerative diseases, have been also found in spinal cord tissue from patients who died of ALS. These processes suggest that inflammation might promote motor neuron death. In addition, in ALS, high CSF levels of glutamate and excitotoxicity have been reported [98]. It has been proved that in ALS, oxidative stress is closely associated with motor neuron degeneration. Several recent clinical researches suggest that there exist a number of biomarkers for oxidative stress in ALS. Mitsumoto et al. [19] have observed that the level of urinary $15-\mathrm{F}_{2 t}$-IsoP and urinary 8 -oxodG was higher among ALS patients than in control. No correlation has been found between age and urinary IsoPs. Protein carbonyl levels did not differ between patients suffering from ALS and controls, in contrast to urinary levels of IsoPs and urinary 8-oxodG, which are strongly correlated. This suggests that $15-\mathrm{F}_{2 t}-\mathrm{IsoPs}$ and 8-oxodG are biomarkers of oxidative stress in patients with ALS. [58, 99].

What is more, it has been proved that the well-established role of COX-2 in inflammation and in glutamate-dependent neurotoxicity is a basic hypothesis of COX-2 involvement in ALS pathogenesis. The increased COX-2 mRNA and protein were found in postmortem spinal cord of ALS patients. Together with the increase of $\mathrm{PGE}_{2}$ tissue levels, the elevated expression of COX-2 has been observed [100]. COX-2 is expressed in neurons in the spinal cord dorsal and ventral horns and also in dorsal root ganglia under normal conditions. The COX-2 expression was markedly evaluated and localized to both neurons and glial cells in postmortem spinal cord of ALS patients. It has been proved that COX-2 is associated with astrocytes and much lesser extent with glial cells [101]. Some studies suggest that inhibition of COX-2 may have therapeutic benefits by altering the cascade of events leading to the progressive neuronal death in ALS patients. But the efficacy of COX-2 inhibition in the presence of overt clinical signs of disease still remains unknown.
In addition, in spinal cords of sporadic ALS patients, the immunoreactivity of 15-deoxy-D12-14-PGJ 2 (15d-PGJ ${ }_{2}$ ) has been found. $15 \mathrm{~d}-\mathrm{PGJ} \mathrm{J}_{2}$, this bioactive prostanoid produced by dehydration and isomerization of $\mathrm{PGD}_{2}$, activates the nuclear peroxisome proliferator-activated receptor $\gamma$ (PPARy). PPARy is a critical transcription factor involved in adipocyte and monocyte differentiation.

This receptor can be considered as a potential therapeutic benefit of its activation in several inflammatory neurological diseases [31].

\section{Conflict of Interests}

The authors declare that there is no conflict of interests regarding the publication of this paper.

\section{References}

[1] A. M. Enciu, M. Gherghiceanu, and B. O. Popescu, "Triggers and effectors of oxidative stress at blood-brain barrier level: relevance for brain ageing and neurodegeneration," Oxidative Medicine and Cellular Longevity, vol. 2013, Article ID 297512, 12 pages, 2013.

[2] E. Niki, "Lipid peroxidation products as oxidative stress biomarkers," BioFactors, vol. 34, no. 2, pp. 171-180, 2008.

[3] R. Santos, C. R. Almodovar, A. L. Bulteau, and C. M. Gomes, "Neurodegeneration, neurogenesis, and oxidative stress," Oxidative Medicine and Cellular Longevity, vol. 2013, Article ID 730581, 2 pages, 2013.

[4] G. Cappellano, M. Carecchio, T. Fleetwood et al., "Immunity and inflammation in neurodegenerative diseases," American Journal of Neurodegenerative Disease, vol. 2, no. 2, pp. 89-107, 2013.

[5] G. Almer, P. Teismann, Z. Stevic et al., "Increased levels of the pro-inflammatory prostaglandin PGE2 in CSF from ALS patients," Neurology, vol. 58, no. 8, pp. 1277-1279, 2002.

[6] T. Farooqui and A. A. Farooqui, "Lipid-mediated oxidative stress and inflammation in the pathogenesis of Parkinson's disease," Parkinson's Disease, vol. 2011, Article ID 247467, 9 pages, 2011.

[7] J. Guest, R. Grant, T. A. Mori, and K. D. Croft, "Changes in oxidative damage, inflammation and $[\operatorname{NAD}(\mathrm{H})]$ with age in cerebrospinal fluid," PLoS ONE, vol. 9, no. 1, Article ID e85335, 2014.

[8] L. J. Roberts II and G. L. Milne, "Isoprostanes," Journal of Lipid Research, vol. 50, pp. S219-S223, 2009.

[9] N. Mattsson, S. Haghighi, O. Andersen et al., "Elevated cerebrospinal fluid $\mathrm{F}_{2}$-isoprostane levels indicating oxidative stress in healthy siblings of multiple sclerosis patients," Neuroscience Letters, vol. 414, no. 3, pp. 233-236, 2007.

[10] L. Minghetti, A. Greco, F. Cardone et al., "Increased brain synthesis of prostaglandin $\mathrm{E}_{2}$ and $\mathrm{F}_{2}$-isoprostane in human and experimental transmissible spongiform encephalopathies," Journal of Neuropathology and Experimental Neurology, vol. 59, no. 10, pp. 866-871, 2000.

[11] T. J. Montine, J. F. Quinn, D. Milatovic et al., "Peripheral $\mathrm{F}_{2}$-isoprostanes and $\mathrm{F}_{4}$-neuroprostanes are not increased in Alzheimer's disease," Annals of Neurology, vol. 52, no. 2, pp. 175-179, 2002.

[12] Y. Yao, V. Zhukareva, S. Sung et al., "Enhanced brain levels of 8,12-iso-iPF $2 \alpha$-VI differentiate AD from frontotemporal dementia," Neurology, vol. 61, no. 4, pp. 475-478, 2003. 
[13] D. Praticò, V. M. Y. Lee, J. Q. Trojanowski, J. Rokach, and G. A. Fitzgerald, "Increased $\mathrm{F}_{2}$-isoprostanes in Alzheimer's disease: evidence for enhanced lipid peroxidation in vivo," FASEB Journal, vol. 12, no. 15, pp. 1777-1783, 1998.

[14] S. E. Browne and M. F. Beal, "Oxidative damage in Huntington's disease pathogenesis," Antioxidants and Redox Signaling, vol. 8, no. 11-12, pp. 2061-2073, 2006.

[15] S. Kralovicova, S. N. Fontaine, A. Alderton et al., "The effects of prion protein expression on metal metabolism," Molecular and Cellular Neuroscience, vol. 41, no. 2, pp. 135-147, 2009.

[16] B. S. Wong, T. Pan, T. Liu et al., "Prion disease: a loss of antioxidant function?" Biochemical and Biophysical Research Communications, vol. 275, no. 2, pp. 249-252, 2000.

[17] L. Minghetti, F. Cardone, A. Greco et al., "Increased CSF levels of prostaglandin $\mathrm{E}_{2}$ in variant Creutzfeldt-Jakob disease," Neurology, vol. 58, no. 1, pp. 127-129, 2002.

[18] S. Arlt, A. Kontush, I. Zerr et al., "Increased lipid peroxidation in cerebrospinal fluid and plasma from patients with creutzfeldtjakob disease," Neurobiology of Disease, vol. 10, no. 2, pp. 150156, 2002.

[19] H. Mitsumoto, R. Santella, X. Liu et al., "Oxidative stress biomarkers in sporadic ALS," Amyotrophic Lateral Sclerosis, vol. 9, no. 3, pp. 177-183, 2008.

[20] R. Zhang, Q. Zhang, J. Niu et al., "Screening of microRNAs associated with Alzheimer's disease using oxidative stress cell model and different strains of senescence accelerated mice," Journal of the Neurological Sciences, vol. 338, pp. 57-64, 2014.

[21] K. D. Jacob, N. Noren, N. Hooten, A. R. Trzeciak, and M. K. Evans, "Markers of oxidant stress that are clinically relevant in aging and age-related disease," Mech Ageing, vol. 134, pp. 139157, 2013.

[22] A. A. Farooqui, L. A. Horrocks, and T. Farooqui, "Interactions between neural membrane glycerophospholipid and sphingolipid mediators: a recipe for neural cell survival or suicide," Journal of Neuroscience Research, vol. 85, no. 9, pp. 1834-1850, 2007.

[23] R. E. Gonsette, "Neurodegeneration in multiple sclerosis: the role of oxidative stress and excitotoxicity," Journal of the Neurological Sciences, vol. 274, no. 1-2, pp. 48-53, 2008.

[24] B. Halliwell and C. Y. J. Lee, "Using isoprostanes as biomarkers of oxidative stress: some rarely considered issues," Antioxidants and Redox Signaling, vol. 13, no. 2, pp. 145-156, 2010.

[25] G. Barrera, "Oxidative stress and lipid peroxidation products in cancer progression and therapy," ISRN Oncology, vol. 2012, Article ID 137289, 21 pages, 2012.

[26] Y. Dotan, D. Lichtenberg, and I. Pinchuk, "Lipid peroxidation cannot be used as a universal criterion of oxidative stress," Progress in Lipid Research, vol. 43, no. 3, pp. 200-227, 2004.

[27] G. L. Milne, H. Yin, K. D. Hardy, S. S. Davies, and L. J. Roberts, "Isoprostane generation and function," Chemical Reviews, vol. 111, no. 10, pp. 5973-5996, 2011.

[28] L. J. Roberts II and J. D. Morrow, "Products of the isoprostane pathway: unique bioactive compounds and markers of lipid peroxidation," Cellular and Molecular Life Sciences, vol. 59, no. 5, pp. 808-820, 2002.

[29] K. D. Hardy, B. E. Cox, G. L. Milne, H. Yin, and L. J. Roberts II, "Nonenzymatic free radical-catalyzed generation of 15-deoxy- $\Delta^{12,14} \mathrm{~J}_{2}$-like compounds (deoxy-J $\mathrm{J}_{2}$-isoprostanes) in vivo1," Journal of Lipid Research, vol. 52, no. 1, pp. 113-124, 2011.

[30] R. C. Murphy and E. Fahy, "Isoprostane nomenclature: more suggestions," Prostaglandins Leukotrienes and Essential Fatty Acids, vol. 82, no. 2-3, pp. 69-70, 2010.
[31] S. G. Harris, J. Padilla, L. Koumas, D. Ray, and R. P. Phipps, "Prostaglandins as modulators of immunity," Trends in Immunology, vol. 23, no. 3, pp. 144-150, 2002.

[32] E. E. Reich, W. R. Markesbery, L. J. Roberts II, L. L. Swift, J. D. Morrow, and T. J. Montine, "Brain regional quantification of F-ring and D-/E-ring isoprostanes and neuroprostanes in Alzheimer's disease," American Journal of Pathology, vol. 158, no. 1, pp. 293-297, 2001.

[33] E. L. Streck, G. A. Czapski, and C. Gonçalves da Silva, "Neurodegeneration, mitochondrial dysfunction, and oxidative stress," Oxidative Medicine and Cellular Longevity, vol. 2013, Article ID 826046, 2 pages, 2013.

[34] Y. Chen, J. D. Morrow, and L. J. Roberts II, "Formation of reactive cyclopentenone compounds in vivo as products of the isoprostane pathway," Journal of Biological Chemistry, vol. 274, no. 16, pp. 10863-10868, 1999.

[35] Y. Chen, W. E. Zackert, L. J. Roberts II, and J. D. Morrow, "Evidence for the formation of a novel cyclopentenone isoprostane, 15-A(2t)-isoprostane (8-iso-prostaglandin A2) in vivo," Biochimica et Biophysica Acta: Molecular and Cell Biology of Lipids, vol. 1436, no. 3, pp. 550-556, 1999.

[36] R. D. O'Brien, "Fats and oils analysis," in Fats and Oils: Formulating and Processing for Applications, pp. 197-260, CRC Press, Boca Raton, Fla, USA, 3rd edition, 2009.

[37] C. de Felice, C. Signorini, T. Durand et al., " $F_{2}$-dihomo-isoprostanes as potential early biomarkers of lipid oxidative damage in Rett syndrome," Journal of Lipid Research, vol. 52, no. 12, pp. 2287-2297, 2011.

[38] A. E. Barden, T. B. Corcoran, E. Mas et al., "Is there a role for isofurans and neuroprostanes in pre-eclampsia and normal pregnancy?" Antioxidants and Redox Signaling, vol. 16, no. 2, pp. 165-169, 2012.

[39] J. D. Brooks, G. L. Milne, H. Yin, S. C. Sanchez, N. A. Porter, and J. D. Morrow, "Formation of highly reactive cyclopentenone isoprostane compounds (A 3/J3-isoprostanes) in vivo from eicosapentaenoic acid," Journal of Biological Chemistry, vol. 283, no. 18, pp. 12043-12055, 2008.

[40] M. Comporti, C. Signorini, B. Arezzini, D. Vecchio, B. Monaco, and C. Gardi, " $F_{2}$-isoprostanes are not just markers of oxidative stress," Free Radical Biology and Medicine, vol. 44, no. 3, pp. 247256, 2008.

[41] S. Zaja-Milatovic, R. C. Gupta, M. Aschner, and D. Milatovic, "Protection of DFP-induced oxidative damage and neurodegeneration by antioxidants and NMDA receptor antagonist," Toxicology and Applied Pharmacology, vol. 240, no. 2, pp. 124131, 2009.

[42] D. Milatovic, T. J. Montine, and M. Aschner, "Measurement of isoprostanes as markers of oxidative stress," Methods in Molecular Biology, vol. 758, pp. 195-204, 2011.

[43] E. D. 'Amico, P. Factor-Litvak, R. M. Santella, and H. Mitsumoto, "Clinical perspective on oxidative stress in sporadic amyotrophic lateral sclerosis," Free Radical Biology \& Medicine, vol. 65, pp. 509-527, 2013.

[44] J. Wong-Ekkabut, Z. Xu, W. Triampo, I. M. Tang, D. P. Tieleman, and L. Monticelli, "Effect of lipid peroxidation on the properties of lipid bilayers: a molecular dynamics study," Biophysical Journal, vol. 93, no. 12, pp. 4225-4236, 2007.

[45] S. Yehuda, S. Rabinovitz, R. L. Carasso, and D. I. Mostofsky, "The role of polyunsaturated fatty acids in restoring the aging neuronal membrane," Neurobiology of Aging, vol. 23, no. 5, pp. 843-853, 2002. 
[46] T. J. Montine, M. D. Neely, J. F. Quinn et al., "Lipid peroxidation in aging brain and Alzheimer's disease," Free Radical Biology and Medicine, vol. 33, no. 5, pp. 620-626, 2002.

[47] E. Miller, A. Walczak, J. Saluk, M. B. Ponczek, and I. Majsterek, "Oxidative modification of patient's plasma proteins and its role in pathogenesis of multiple sclerosis," Clinical Biochemistry, vol. 45, no. 1-2, pp. 26-30, 2012.

[48] Z. Korade, L. Xu, K. Mirnics, and N. A. Porter, "Lipid biomarkers of oxidative stress in a genetic mouse model of Smith-LemliOpitz syndrome," Journal of Inherited Metabolic Disease, vol. 36, no. 1, pp. 113-122, 2013.

[49] G. L. Milne, B. Gao, E. S. Terry, W. E. Zackert, and S. C. Sanchez, "Measurement of $\mathrm{F}_{2}$-isoprostanes and isofurans using gas chromatography-mass spectrometry," Free Radical Biology \& Medicine, vol. 59, pp. 36-44, 2013.

[50] B. Casetta, M. Longini, F. Proietti, S. Perrone, and G. Buonocore, "Development of a fast and simple LC-MS/MS method for measuring the $\mathrm{F}_{2}$-isoprostanes in newborns," Journal of MaternalFetal and Neonatal Medicine, vol. 25, no. 1, pp. 114-118, 2012.

[51] G. L. Milne, H. Yin, and J. D. Morrow, "Human biochemistry of the isoprostane pathway," Journal of Biological Chemistry, vol. 283, no. 23, pp. 15533-15537, 2008.

[52] G. L. Milne, H. Yin, K. D. Hardy, S. S. Davies, and L. J. Roberts, "Isoprostane generation andfunction," Chemical Reviews, vol. 111, no. 10, pp. 5973-5996, 2011.

[53] S. Bleich, S. Kropp, D. Degner et al., "Creutzfeldt-Jakob disease and oxidative stress," Acta Neurologica Scandinavica, vol. 101, no. 5, pp. 332-334, 2000.

[54] T. J. Montine, M. F. Beal, D. Robertson et al., "Cerebrospinal fluid $\mathrm{F}_{2}$-isoprostanes are elevated in Huntington's disease," Neurology, vol. 52, no. 5, pp. 1104-1105, 1999.

[55] E. Miller, M. Mrowicka, J. Saluk-Juszczak, and M. Ireneusz, "The level of isoprostanes as a non-invasive marker for in vivo lipid peroxidation in secondary progressive multiple sclerosis," Neurochemical Research, vol. 36, no. 6, pp. 1012-1016, 2011.

[56] L. Minghetti, F. Cardone, A. Greco et al., "Increased CSF levels of prostaglandin $\mathrm{E}_{2}$ in variant Creutzfeldt-Jakob disease," Neurology, vol. 58, no. 1, pp. 127-129, 2002.

[57] D. T. Walsh, V. H. Perry, and L. Minghetti, "Cyclooxygenase-2 is highly expressed in microglial-like cells in a murine model of prion disease," Glia, vol. 29, pp. 392-396, 2000.

[58] C. Consilvio, A. M. Vincent, and E. L. Feldman, "Neuroinflammation, COX-2, and ALS-a dual role?" Experimental Neurology, vol. 187, no. 1, pp. 1-10, 2004.

[59] L. J. Roberts II, J. P. Fessel, and S. S. Davies, "The biochemistry of the isoprostane, neuroprostane, and isofuran pathways of lipid peroxidation," Brain Pathology, vol. 15, no. 2, pp. 143-148, 2005.

[60] D. Milatovic and M. Aschner, "Measurement of isoprostanes as markers of oxidative stress in neuronal tissue," in Current Protocols in Toxicology, unit 12.14, pp. 1-12, 2009.

[61] A. Melo, L. Monteiro, R. M. F. Lima, D. M. de Oliveira, M. D. de Cerqueira, and R. S. El-Bachá, "Oxidative stress in neurodegenerative diseases: mechanisms and therapeutic perspectives," Oxidative Medicine and Cellular Longevity, vol. 2011, Article ID 467180, 14 pages, 2011.

[62] G. G. Ortiz, F. P. Pacheco-Moisés, O. K. Bitzer-Quintero et al., "Immunology and oxidative stress in multiple sclerosis: clinical and basic approach," Journal of Cerebral Blood Flow \& Metabolism, vol. 34, pp. 34-42, 2014.

[63] R. Sultana and D. A. Butterfield, "Role of oxidative stress in the progression of Alzheimer's disease," Journal of Alzheimer's Disease, vol. 19, no. 1, pp. 341-353, 2010.
[64] S. Ayala-Peña, "Role of oxidative DNA damage in mitochondrial dysfunction and Huntington's disease pathogenesis," Free Radical Biology \& Medicine, vol. 62, pp. 102-110, 2013.

[65] D. A. Butterfield, M. L. Bader Lange, and R. Sultana, "Involvements of the lipid peroxidation product, HNE, in the pathogenesis and progression of Alzheimer's disease," Biochimica et Biophysica Acta: Molecular and Cell Biology of Lipids, vol. 1801, no. 8, pp. 924-929, 2010.

[66] A. Greco, L. Minghetti, and G. Levi, "Isoprostanes, novel markers of oxidative injury, help understanding the pathogenesis of neurodegenerative diseases," Neurochemical Research, vol. 25, no. 9-10, pp. 1357-1364, 2000.

[67] E. Miller, "Neurodegenerative diseases," Advances in Experimental Medicine and Biology, vol. 724, pp. 228-238, 2012.

[68] D. Karussis, "The diagnosis of multiple sclerosis and the various related demyelinating syndromes: a critical review," Journal of Autoimmunity, vol. 48-49, pp. 134-142, 2014.

[69] S. Gandhi and A. Y. Abramov, "Mechanism of oxidative stress in neurodegeneration," Oxidative Medicine and Cellular Longevity, vol. 2012, Article ID 428010, 11 pages, 2012.

[70] L. K. Peterson and R. S. Fujinami, "Inflammation, demyelination, neurodegeneration and neuroprotection in the pathogenesis of multiple sclerosis," Journal of Neuroimmunology, vol. 184, no. 1-2, pp. 37-44, 2007.

[71] E. Miller, M. Mrowicka, K. Malinowska, J. Mrowicki, J. SalukJuszczak, and J. Kędziora, "Effects of whole-body cryotherapy on a total antioxidative status and activities of antioxidative enzymes in blood of depressive multiple sclerosis patients," World Journal of Biological Psychiatry, vol. 12, no. 3, pp. 223-227, 2011.

[72] S. Ljubisavljevic, I. Stojanovic, D. Pavlovic et al., "Suppression of the lipid peroxidation process in the CNS reduces neurological expression of experimentally induced autoimmune encephalomyelitis," Folia Neuropathologica, vol. 51, pp. 51-57, 2013.

[73] A. Greco and L. Minghetti, "Isoprostanes as biomarkers and mediators of oxidative injury in infant and adult central nervous system diseases," Current Neurovascular Research, vol. 1, no. 4, pp. 341-354, 2004.

[74] S. Mondragón-Rodríguez, G. Perry, X. Zhu, and J. Boehm, "Amyloid beta and tau proteins as therapeutic targets for Alzheimer's disease treatment: rethinking the current strategy," International Journal of Alzheimer's Disease, vol. 2012, Article ID 630182, 7 pages, 2012.

[75] M. G. Savelieff, S. Lee, Y. Liu, and M. H. Lim, "Untangling amyloid- $\beta$, tau, and metals in Alzheimer's disease," ACS Chemical Biology, vol. 8, pp. 856-865, 2013.

[76] G. Ellis, E. Fang, M. Maheshwari et al., "Lipid oxidation and modification of amyloid- $\beta(\mathrm{A} \beta)$ in vitro and in vivo," Journal of Alzheimer's Disease, vol. 22, no. 2, pp. 593-607, 2010.

[77] R. Sultana, M. Perluigi, and A. D. Butterfield, "Lipid peroxidation triggers neurodegeneration: a redox proteomics view into the Alzheimer disease brain," Free Radical Biology \& Medicine, vol. 62, pp. 157-169, 2013.

[78] S. M. Pritchard, P. J. Dolan, A. Vitkus, and G. V. W. Johnson, "The toxicity of tau in Alzheimer disease: turnover, targets and potential therapeutics," Journal of Cellular and Molecular Medicine, vol. 15, no. 8, pp. 1621-1635, 2011.

[79] Y. Zhao and B. Zhao, "Oxidative stress and the pathogenesis of Alzheimer's disease," Oxidative Medicine and Cellular Longevity, vol. 2013, Article ID 316523, 10 pages, 2013. 
[80] E. S. Musiek, J. K. Cha, H. Yin et al., "Quantification of F-ring isoprostane-like compounds ( $\mathrm{F}_{4}$-neuroprostanes) derived from docosahexaenoic acid in vivo in humans by a stable isotope dilution mass spectrometric assay," Journal of Chromatography B: Analytical Technologies in the Biomedical and Life Sciences, vol. 799, no. 1, pp. 95-102, 2004.

[81] J. Long, P. He, Y. Shen, and R. Li, "New evidence of mitochondria dysfunction in the female Alzheimer's disease brain: deficiency of estrogen receptor- $\beta$,' Journal of Alzheimer's Disease, vol. 30, pp. 545-558, 2012.

[82] A. Mancini, S. Raimondo, M. Persano et al., "Estrogens as antioxidant modulators in human fertility," International Journal of Endocrinology, vol. 2013, Article ID 607939, 6 pages, 2013.

[83] D. Praticò, J. Rokach, and R. K. Tangirala, "Brains of aged apolipoprotein E-deficient mice have increased levels of $\mathrm{F}_{2}$-isoprostanes, in vivo markers of lipid peroxidation," Journal of Neurochemistry, vol. 73, no. 2, pp. 736-741, 1999.

[84] F. Sánchez-López, I. Tasset, E. Agüera et al., "Oxidative stress and inflammation biomarkers in the blood of patients with Huntington's disease," Neurological Research, vol. 34, pp. 721724, 2012.

[85] M. A. Sorolla, G. Reverter-Branchat, J. Tamarit, I. Ferrer, J. Ros, and E. Cabiscol, "Proteomic and oxidative stress analysis in human brain samples of Huntington disease," Free Radical Biology and Medicine, vol. 45, no. 5, pp. 667-678, 2008.

[86] S. E. Browne and M. F. Beal, "Oxidative damage in Huntington's disease pathogenesis," Antioxidants and Redox Signaling, vol. 8, no. 11-12, pp. 2061-2073, 2006.

[87] F. Haun, T. Nakamura, and S. A. Lipton, "Dysfunctional mitochondrial dynamics in the pathophysiology of neurodegenerative diseases," Journal of Cell Death, vol. 6, pp. 27-35, 2013.

[88] E. C. Stack, W. R. Matson, and R. J. Ferrante, "Evidence of oxidant damage in Huntington's disease: translational strategies using antioxidants," Annals of the New York Academy of Sciences, vol. 1147, pp. 79-92, 2008.

[89] L. Gan and J. A. Johnson, "Oxidative damage and the Nrf2-ARE pathway in neurodegenerative diseases," Biochimica et Biophysica Acta, 2013.

[90] A. Carrizzo, A. Di Pardo, V. Maglione et al., "Nitric oxide dysregulation in platelets from patients with advanced huntington disease," PLoS ONE, vol. 9, no. 2, Article ID e89745, 2014.

[91] N. Stoy, G. M. Mackay, C. M. Forrest et al., “Tryptophan metabolism and oxidative stress in patients with Huntington's disease," Journal of Neurochemistry, vol. 93, no. 3, pp. 611-623, 2005.

[92] N. Klepac, M. Relja, R. Klepac, S. Hećimović, T. Babić, and V. Trkulja, "Oxidative stress parameters in plasma of Huntington's disease patients, asymptomatic Huntington's disease gene carriers and healthy subjects: a cross-sectional study," Journal of Neurology, vol. 254, no. 12, pp. 1676-1683, 2007.

[93] B. S. Wong, T. Liu, R. Li et al., "Increased levels of oxidative stress markers detected in the brains of mice devoid of prion protein," Journal of Neurochemistry, vol. 76, no. 2, pp. 565-572, 2001.

[94] M. Guentchev, T. Voigtländer, C. Haberler, M. H. Groschup, and H. Budka, "Evidence for oxidative stress in experimental prion disease," Neurobiology of Disease, vol. 7, no. 4, pp. 270-273, 2000.

[95] B. S. Wong, D. R. Brown, T. Pan et al., "Oxidative impairment in scrapie-infected mice is associated with brain metals perturbations and altered antioxidant activities," Journal of Neurochemistry, vol. 79, no. 3, pp. 689-698, 2001.

[96] C. Bate, S. Rutherford, M. Gravenor, S. Reid, and A. Williams, "Cyclo-oxygenase inhibitors protect against prion-induced neurotoxicity in vitro," NeuroReport, vol. 13, no. 15, pp. $1933-$ 1938, 2002.

[97] S. Boillée, C. Vande Velde, and D. Cleveland, "ALS: a disease of motor neurons and their nonneuronal neighbors," Neuron, vol. 52, no. 1, pp. 39-59, 2006.

[98] P. F. Pradat, S. Attarian, J. P. Camdessanché et al., "Research in amyotrophic lateral sclerosis: what is new in 2009?" Revue Neurologique, vol. 166, no. 8-9, pp. 683-698, 2010.

[99] H. Mitsumoto, R. Santella, X. Liu et al., "Oxidative stress biomarkers in sporadic ALS," Amyotrophic Lateral Sclerosis, vol. 9, no. 3, pp. 177-183, 2008.

[100] S. C. Barber and P. J. Shaw, "Oxidative stress in ALS: key role in motor neuron injury and therapeutic target," Free Radical Biology and Medicine, vol. 48, no. 5, pp. 629-641, 2010.

[101] G. Almer, C. Guegan, P. Teismann et al., "Increased expression of the pro-inflammatory enzyme cyclooxygenase- 2 in amyotrophic lateral sclerosis," Annals of Neurology, vol. 49, no. 2, pp. 176-185, 2001. 


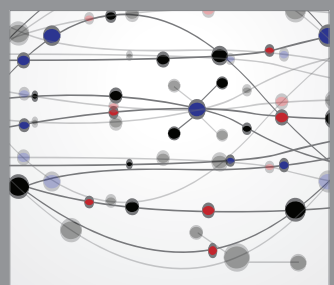

The Scientific World Journal
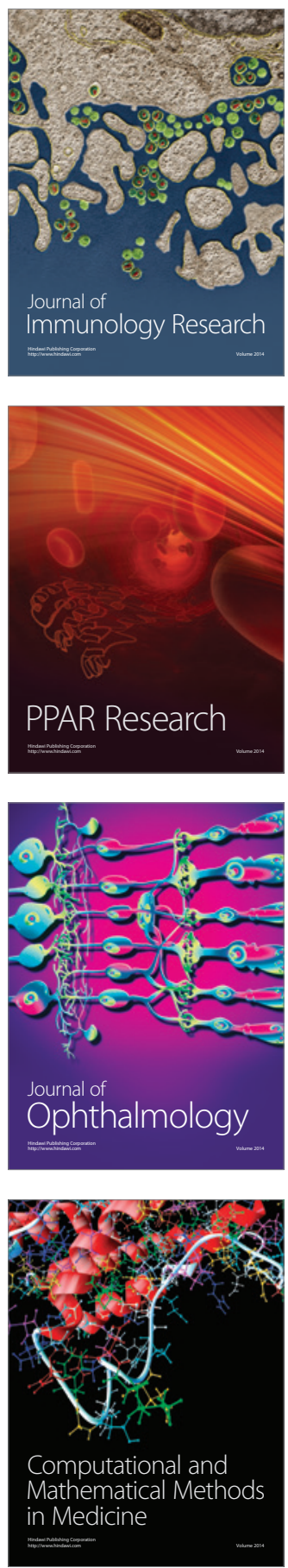

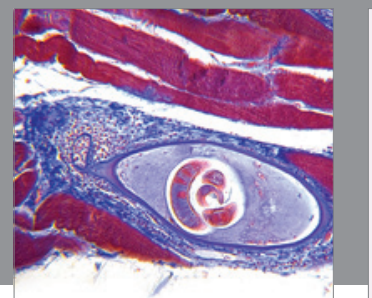

Gastroenterology

Research and Practice
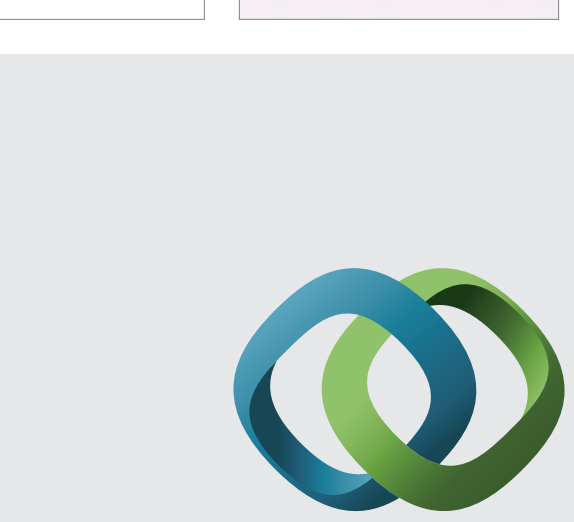

\section{Hindawi}

Submit your manuscripts at

http://www.hindawi.com

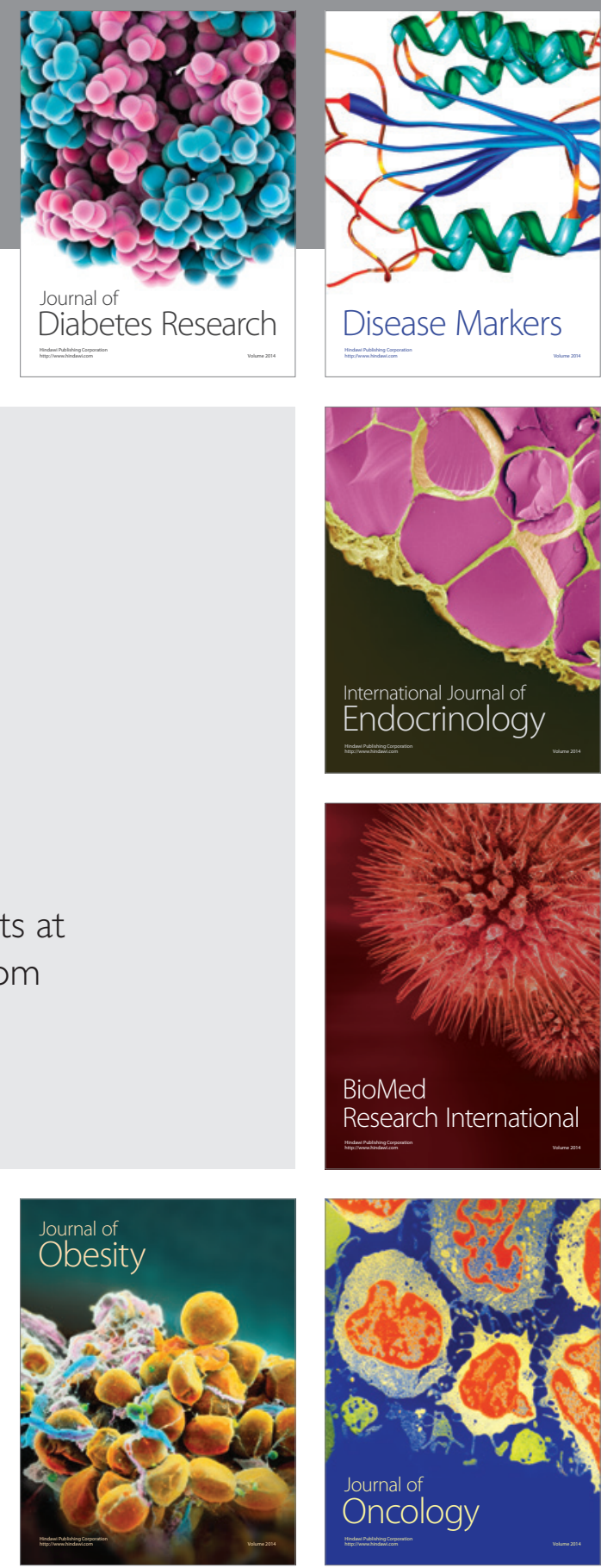

Disease Markers
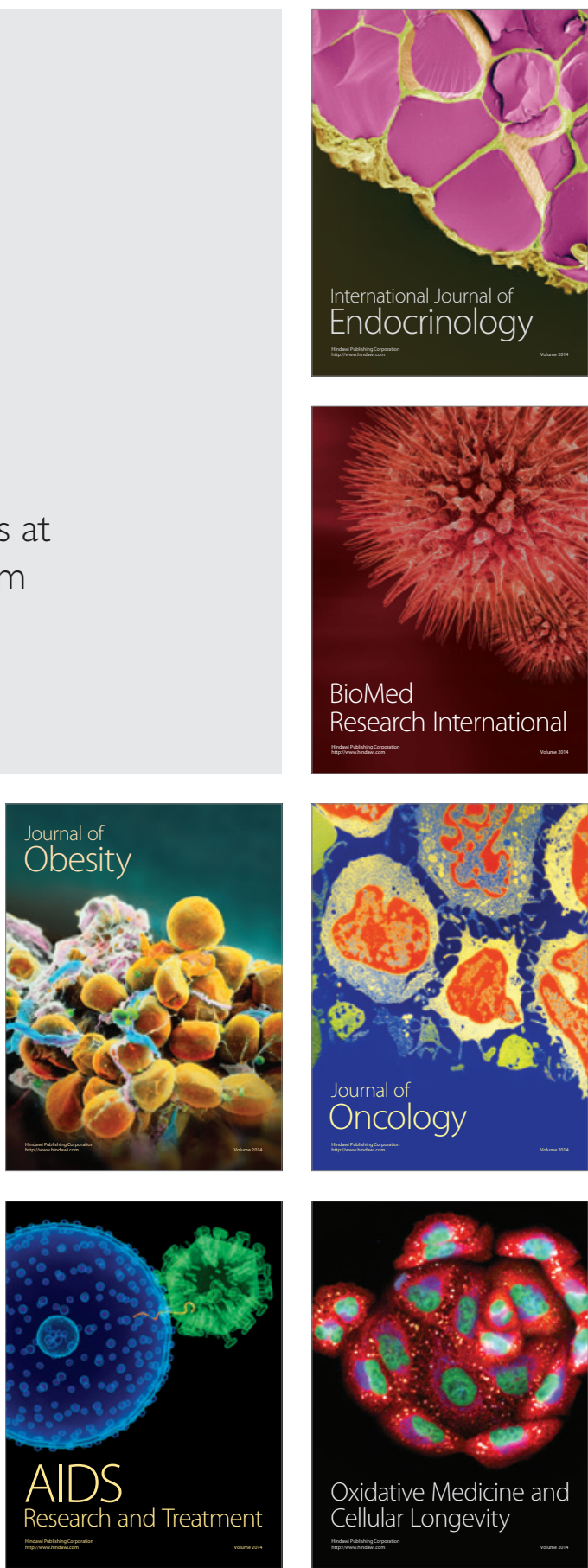